\title{
When Ising meets Majorana
}

The topological degeneracy associated with Majorana edge states has been measured in a spin-1/2 chain of cobalt atoms, thereby opening new avenues in low-dimensional quantum magnetism.

\section{Frédéric Mila}

$\mathrm{T}$ opological states of matter have attracted significant attention because their potential for quantum computing. These often consist of edge states that correspond to emergent degrees of freedom that are distinct from those in the bulk. A few years ago, it was shown that a simple one-dimensional model of a quantum wire coupled to a superconductor can support two Majorana edge states, whereas all other excitations in the system are fermionic in character ${ }^{1}$. Unlike standard fermions, Majorana fermions are their own antiparticle, but two Majorana fermions can be recombined to give rise to a standard fermionic state, which can be either empty or occupied. As a consequence of this, the ground state of a system with Majorana edge excitations is expected to be two-fold degenerate.

Although there has been significant progress towards the detection of Majorana edge states in quantum wires ${ }^{2,3}$, the same physics is present in the paradigmatic model for quantum phase transitions, the transverse field Ising model ${ }^{4}$. While this model can be rigorously mapped onto a fermionic model with superconducting pairing, it has received comparatively less experimental attention. Writing in Nature Physics, R. Toskovic and collaborators ${ }^{5}$ now report clear evidence in favour of two quasi-degenerate ground states typical of Majorana edge states in atomic chains of cobalt.

Although cobalt atoms carry a spin of $3 / 2$, they are effectively spin- $1 / 2$ because of the presence of an easy-plane anisotropy. Under an applied external field, a chain of cobalt atoms is thus described by a variant of the transverse field Ising model with an additional Ising coupling along the field. The key advantage of this model over the standard transverse field Ising model is that, in a finite chain, the two low-lying states, which can be distinguished by the parity of the number of up spins, cross each other several times with increasing field ${ }^{6}$. Therefore, although the two ground states would be very difficult to distinguish by any local probe, keeping track of their crossings proves that there must be two of them. To

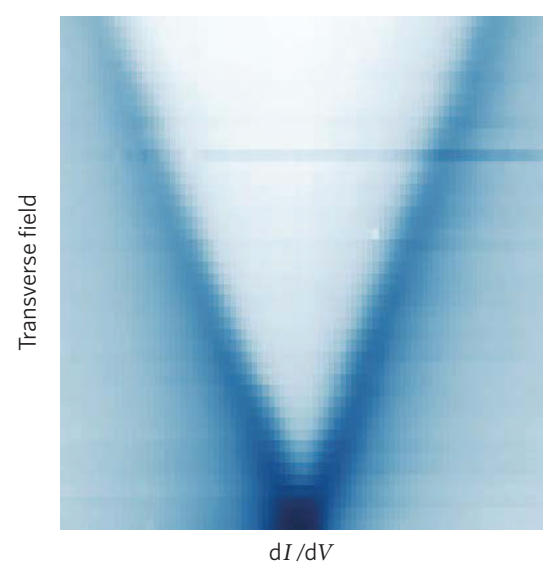

Figure 1 | Characteristic V-shape of the differential conductance of a single cobalt atom when plotted with the transverse field on the vertical axis ${ }^{5}$.

detect these level crossings, Toskovic and colleagues employed scanning tunnelling microscopy to build chains of up to nine cobalt atoms on a metallic substrate with an insulating buffer layer, and then measured the differential conductance separately at each site of the chains as a function of the external field. By keeping track of all singularities at all sites, the authors found evidence of all the expected level crossings - an impressive achievement.

The implications of these level crossings are far reaching. As the authors point out, the level crossings become very dense when approaching the quantum phase transition, which demonstrates the feasibility of experimentally accessing the finite-size effects close to a quantum phase transition. These are well-known in numerical simulations, but are typically much harder to study systematically in experiment. But perhaps more importantly, the very fact that level crossings themselves have been observed is a clear indication that there must be two quasi-degenerate states, as it should if there are Majorana edge states. A similar experiment has recently been proposed to establish the quasi-degeneracy of a quantum wire coupled to a superconductor, by keeping track of the level crossings between sectors with different electron number parity when monitoring, for example, the chemical potential? .
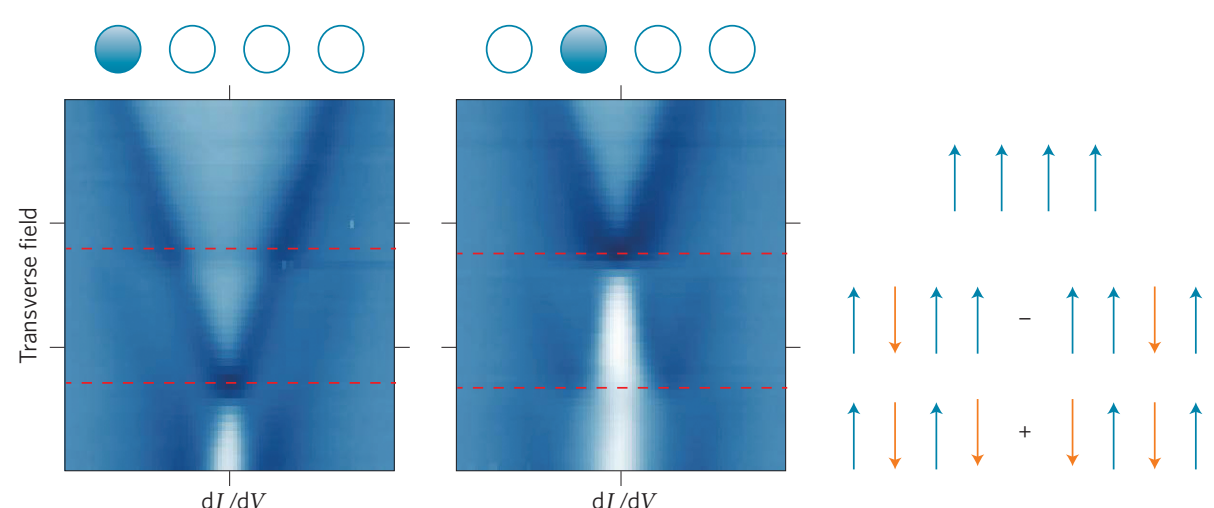

Figure 2 | Differential conductance of a four-site chain versus transverse field recorded at the first (left) and second (middle) sites ${ }^{5}$. The dashed red lines indicate the field values at which level crossings are expected to occur. The right panel shows the approximate ground state wavefunction in each phase. The quantization axis is chosen along the field, and at each transition the parity of the number of up spins must change by one. The data are fully consistent with these wavefunctions: at high field, the state is fully polarized. At the first transition, one spin is flipped. Because the transition is visible at the second site but not the first, the spin must be flipped at the second site, which is in agreement with the intermediate wavefunction. At the second transition, a spin must be flipped at the edge because the transition is visible at the first site and not the second, which is in agreement with the bottom wavefunction. 
Beyond topological edge states, this work is a significant step forwards in the investigation of low-dimensional quantum magnetism for magnetic adatoms. In a ground-breaking paper in 2012, Khajetoorians et al. built chains and small clusters of iron adatoms and showed that the local magnetization at each site can be accessed with spin-resolved scanning tunnelling microscopy ${ }^{8}$. Owing to the strong easy-axis anisotropy of the iron atoms, this work paved the way towards investigating the Heisenberg model with strong Ising anisotropy. In contrast, cobalt atoms have easy-plane anisotropy, which leads to effective models with XY symmetry. The work of Toskovic et al. therefore opens the way to the investigation of a completely different class of quantum magnets.

However, before this kind of approach can be turned into a systematic way of investigating the quantum XY model, it will be important to make progress on the theory of the differential conductance in clusters of cobalt atoms. The differential conductance of an isolated cobalt atom is dominated at low energy by a narrow peak known as the
Kondo resonance ${ }^{9}$, a direct consequence of the two scattering states available for the effective spin-1/2. This resonance splits in the presence of a field, leading to a characteristic V-shape (Fig. 1). What happens when cobalt atoms are coupled by exchange interactions? As acknowledged by Toskovic and colleagues, this presents a theoretical challenge, as the cobalt atoms form a Kondo lattice - a notoriously difficult problem.

Personally, I am confident that simple ideas can be tested in order to attack this issue in cobalt chains. Here is one suggestion: for a chain of cobalt atoms, the conditions for a Kondo resonance can be expected to be satisfied at each level crossing and, to a first approximation, a series of V-shaped anomalies starting at each level crossing can be expected to appear. However, there is an important subtlety to consider: the amplitude of the Kondo resonance is proportional to the matrix elements of the local spin operator between the two states that cross. This amplitude will be very small if the local spin is unaffected by the crossing, leading to different spectra at different sites. In turn, this provides a straightforward interpretation of the data for four sites (Fig. 2). Whether simple ideas of this kind can be backed up by more rigorous approaches and developed into a full theory is an open question. In any case, the possibility of making a rigorous connection between experiments and subtle theoretical problems on finite clusters with precisely defined geometries, such as the Kondo lattice or the transverse field Ising model, is a very exciting development.

Frédéric Mila is at the Institute of Physics, École Polytechnique Fédérale de Lausanne, 1015 Lausanne, Switzerland. e-mail: frederic.mila@epfl.ch

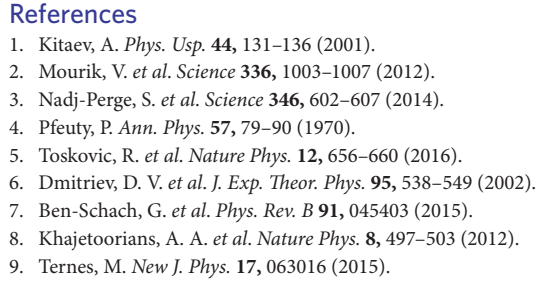

Published online: 18 April 2016

\section{QUANTUM DOTS}

\section{Nuclear spins get a comb-over}

A frequency comb technique used in NMR spectroscopy reveals the dynamics of the nuclear spin bath in selfassembled quantum dots.

\section{Jeroen Elzerman and Mark Buitelaar}

$\mathrm{N}$ uclear magnetic resonance $(\mathrm{NMR})$ is an extremely versatile spectroscopic technique that underpins applications ranging from the structural characterization of molecules to medical imaging. In recent years, optically detected NMR has been used to study self-assembled quantum dots ${ }^{1,2}$, which are of interest as sources of single photons and entangled photon pairs ${ }^{3}$, as well as for quantum networks. ${ }^{4}$ These dots are highly strained, leading to a large inhomogeneous broadening of the NMR spectrum that masks the subtle dynamics of the nuclear spins. Writing in Nature Physics, Andreas Waeber and colleagues ${ }^{5}$ demonstrate an NMR technique, based on frequency combs in the radiofrequency (RF) regime, that is capable of probing the ultraslow fluctuations of the nuclear spin bath. Their measurements reveal that the correlation time of the nuclear spin fluctuations can be of the order of seconds at low temperature and high magnetic field.
Self-assembled quantum dots are nanoscale islands of InAs grown epitaxially inside GaAs. These dots can host electron or hole spins that can be manipulated and probed optically, which allows them to be used as spin qubits. However, the coherence of an electron spin in a self-assembled dot is limited by the hyperfine interaction with the $\sim 10^{5}$ nuclear spins that comprise the dot. This 'central spin problem' is also important for other types of spin qubits, such as colour centres in diamond ${ }^{6}$, donors in semiconductors ${ }^{7}$ and gate-defined quantum dots $^{8}$. The hope is that by understanding the dynamics of the nuclear spins in detail, we might find ways to control them and thereby enhance the coherence times of spin qubits.

The nuclear spins in a self-assembled quantum dot experience a very rich environment, which includes dipolar interactions with nearby nuclear spins in the bath and the hyperfine interaction with an electron or hole inside the dot. Strain, owing to the lattice mismatch between InAs and GaAs, adds a further complication whose importance has only recently been appreciated. The strain in self-assembled quantum dots is highly inhomogeneous (Fig. 1). This means each nuclear spin feels a different local electric-field gradient, leading to a position-dependent shift in its NMR resonances through the quadrupolar interaction. The net result is a large inhomogeneous broadening of the dot's NMR spectrum, which masks the much smaller homogeneous linewidths of the individual nuclei.

Conventional pulsed-NMR tries to eliminate inhomogeneous broadening by using spin echos. However, to do this for the full inhomogeneously broadened spectrum in self-assembled quantum dots would require RF powers that are not experimentally feasible and would result in unwanted spin dynamics. To overcome this problem, Waeber and colleagues turned to a tool known from quantum optics: the frequency comb. They first optically polarized the nuclear spins 